\title{
Morphology Changes in Bulk Donor-Acceptor Poly(Benzodithiophene-Benzotriazole) after Post-Treatment
}

Wojciech Zajaczkowski ${ }^{1}$, Tomasz Marszalek ${ }^{1}$, Wallace W. H. Wong ${ }^{3}$, Wojciech Pisula ${ }^{1,2, *}$

${ }^{1}$ Max Planck Institute for Polymer Research, Ackermannweg 10, D-55128 Mainz, Germany

2 Department of Molecular Physics, Lodz University of Technology, Zeromskiego 116, 90-924

Lodz, Poland

${ }^{3}$ School of Chemistry, Bio21 Institute, University of Melbourne, 30 Flemington Road, Parkville, Australia

*Corresponding author: pisula@ mpip-mainz.mpg.de

\begin{abstract}
The bulk order in donor-acceptor semi-amorphous poly(benzodithiophene-benzotriazole) was improved by two different post-treatment procedures applied to the specimen. Two-dimensional wide-angle $\mathrm{X}$-ray scattering was used to investigate the structural changes after treatment. After post-treatment the polymer turned into a highly crystalline morphology with well-resolved and intensive $\pi$-stacking reflections which were absent in the pristine sample. To understand the ordering mechanisms taking place during the two post-treatment procedures, structural parameters like the coherence length and paracrystalinity were extracted from the X-ray data indicating the impact on crystallite size and cumulative lattice disorder. During temperature annealing the intralayer packing transforms from amorphous to highly ordered. On the other hand, solvent annealing enhances mainly the interlayer organization due to interpenetration of solvent molecules into the alkyl mantel. These results provide important insights for the morphology optimization of semi-amorphous conjugated polymers.
\end{abstract}

\section{Introduction}

Conjugated polymers are attractive as semiconducting materials for novel electronic technologies in the fields of solar cells and field-effect transistors (FETs). ${ }^{1}$ In comparison to traditional inorganic semiconductors, conjugated polymers can be structurally modified to tune their solubility allowing their thin film processing from organic solvents. ${ }^{2}$ Moreover, conjugated polymers are mechanically bendable opening the door towards flexible organic electronics. Among this class of materials, donor-acceptor (D-A) polymers have gain particular attention. ${ }^{3}$ The backbone of these polymers consists of an alternating array of electron-rich, donor, or 
electron-deficient, acceptor, moieties. By using appropriate repeat units, the UV-vis absorption profile and the frontier orbital energy gap can be tuned. ${ }^{4}$ This is a particularly important material property for solar cell applications. ${ }^{5}$ Recently, these polymers became also important for transistors since their supramolecular packing can be improved by tailoring the polymer structure yielding close $\pi$-stacking distances, high crystallinity and finally record charge carrier mobilities. ${ }^{6}$ Additionally, the relative arrangement of the donor and acceptor units between neighbouring polymer chains plays a role on the orbital overlap and thus on the type of transported charge carriers. ${ }^{7}$ One main tool for controlling the polymer packing and intralayer arrangement are the substitution position and geometry of solubilizing alkyl chains. ${ }^{8}$ The selfassembly into distinct superstructures depends on the balance between attractive $\pi$-stacking interactions between conjugated backbones and sterically triggered repulsive forces of the alkyl side chains. Interestingly, several reported poorly ordered D-A polymers revealed a high performance either in solar cells or in transistors. ${ }^{9}$ These semi-amorphous polymers carrying typically branched alkyl chains are ordered mainly in the interlayer crystallographic direction, but are poorly packed with disordered $\pi$-stacking. This seemingly contradicting behaviour was attributed to the ability of the polymers to form aggregates as a function of polymer molecular weight. ${ }^{10}$ The established short-range order permits interconnection between aggregates and generates local pathways for the charge carries leading to a high device performance. The main challenge in optimizing semi-amorphous polymeric semiconductors is the relation between polymer structure, morphology and device properties.

In this work, donor-acceptor poly(benzodithiophene-benzotriazole) (Scheme 1, BDT-BTZ) is used as a model system for semi-amorphous polymers. Both units are popular building blocks for conjugated D-A polymers attractive for solar cells and FETs. ${ }^{11}$ Bulk heterojunction solar cells based on BDT-BTZ as an electron donor and $\mathrm{PC}_{71} \mathrm{BM}$ as electron acceptor in an inverted device structure yielded a power-conversion efficiency of $3.45 \%$, while hole mobilities of about $10^{-4}$ $\mathrm{cm}^{2} / \mathrm{Vs}$ were determined for the pure polymer in FETs. ${ }^{12}$ To improve the crystallinity and especially the intralayer packing from amorphous to high order along the $\pi$-stacking direction, the bulk polymer was exposed to two different post-treatments based on temperature and solvent annealing. Instead of thin films, bulk samples were investigated by X-ray scattering to avoid surface ordering effects. After post-treatment the polymer turned into a highly crystalline morphology with well-resolved and intensive $\pi$-stacking reflections which were absent in the 
pristine samples. Relevant parameters concerning the morphology were extracted from the structural data indicating different ordering mechanisms taking place during the two posttreatment procedures. While during temperature annealing particular the polymer packing in the layer structure is improved, solvent annealing enhances mainly the interlayer order due to penetration of solvent molecules between side chains. These results provide important insights into the mechanism of order improvement in initially semi-amorphous conjugated D-A polymers and might be essential for the morphology optimization in thin film devices.

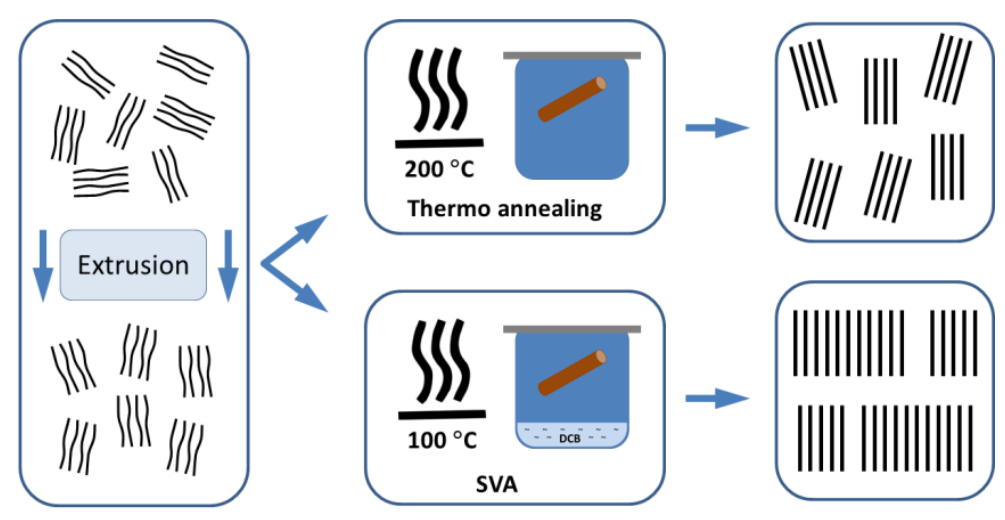

Figure 1. Schematic illustration of the polymer morphology after fiber extrusion and the posttreatment procedures.

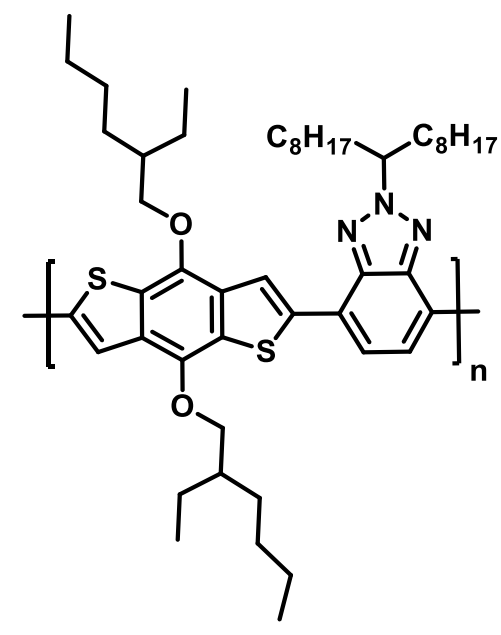

Scheme 1. Chemical structure of BDT-BTZ.

\section{Results and discussion}


Thin films of organic materials, particularly conjugated polymers, fabricated from solution by spin coating or drop casting often contain large areas with kinetically frozen states where shortrange order and pronounced disorder is present. Thermal annealing is as an effective technique for improving the organization and order of the molecules in the film. However, high temperature and long annealing times can lead to molecular degradation. Solvent vapor annealing (SVA) has been proven as an effective alternative and yields ordered structures that are unattainable through thermal annealing. ${ }^{12-13}$ During this annealing procedure taking place at lower temperatures, solvent vapor penetrates into the thin film initiating a local re-organization of the molecules increasing the crystallinity of e.g. polymers ${ }^{14}$, oligomers ${ }^{15}$ or small molecules ${ }^{16}$ and tuning the morphology into a layer with well-ordered structures. To improve the order in semi-amorphous polymers, both treatments were applied on bulk samples in order to avoid surface effects and the structural changes were studied by X-ray scattering.

To determinate the bulk structure of the BDT-BTZ polymer samples, two-dimensional wideangle X-ray scattering (2DWAXS) measurements were performed. The samples were prepared by fiber extrusion and vertically mounted in front of the $2 \mathrm{D}$ detector. The extrusion process induces a macroscopic orientation of the polymer chains by mechanical shear forces in the specimen (Figure 1). Due to their high molecular aspect ratio, the backbones typically oriented along the shearing direction and thus along the length of the fiber. In contrast to the BraggBrentano theta-theta configuration, 2DWAXS provides a direct insight into the polymer arrangement within the corresponding superstructure. This is possible since one of the lattice axes is oriented along the fiber and therefore well-defined for the data analysis. The two other lattice axes are statistically distributed perpendicular around the fiber axis. For the analysis, the recorded patterns are separated into two main planes with equatorial ( $\mathrm{q}_{\mathrm{xy}}$, along fiber thickness) and meridional $\left(\mathrm{q}_{\mathrm{z}}\right.$, along fiber axis) directions on which most of the elliptical reflections are located. ${ }^{17}$ Figure 2 shows three representative 2DWAXS patterns for BDT-BTZ recorded at room temperature before and after applying the post-treatment procedures (Figure 1). All samples exhibit a characteristic distribution of reflections located on the equatorial and meridional planes confirming the typical polymer alignment for BDT-BTZ in the extrusion direction. ${ }^{18}$ Differences in the distribution of reflections between the pristine sample $\mathbf{S 1}$, thermal annealed S2, and solvent annealed S3, showed changes in polymer order before and after posttreatment (Figure 1). Pristine sample S1 exhibited pronounced disorder in bulk as suggested by 
lack of distinct scattering intensities in both small- and wide-angle range (Figure 2a). The broad equatorial reflection in the small-angle range with its maxima located at $q_{\mathrm{xy}}=0.375 \AA^{-1}$ corresponds to the $\mathrm{d}$-spacing of $\mathrm{d}_{100}=1.68 \mathrm{~nm}$ which is assigned, according to Miller's index, to the crystallographic axis of (h00). Additionally, low intensity reflections on the same plane at $q_{\mathrm{xy}}=0.553 \AA^{-1}$ are attributed to the d-spacing of $\mathrm{d}_{010}=1.14 \mathrm{~nm}$ along the $(0 \mathrm{k} 0)$ crystallographic direction. The broad scattering reflections in wide-angle range between $q_{\mathrm{xy}}=1.20-1.50 \AA^{-1}(\mathrm{~d} \approx$ $0.52-0.42 \mathrm{~nm}$ ) are composed of the superposition of scattering intensities originating from the $\pi$-stacking period, higher order reflections of the interlayer spacing and the isotropic "halo" of the disordered alkyl chains. The absence of distinct reflections in this scattering range confirms once again strong inter- and intra-lamellar disorder. The middle-angle region on the meridional plane displays strong and sharp scattering intensities at $q_{z}=1.00 \AA^{-1}\left(\mathrm{~d}_{002}=0.63 \mathrm{~nm}\right)$ which are assigned as $2^{\text {nd }}$ order reflections related to the length of the single repeat polymer unit ( $\mathrm{d}_{001}=1.26$ $\mathrm{nm}$ ) and arranged parallel to the crystallographic direction of (001). In comparison to sample $\mathbf{S 1}$, the samples after post-treatment, $\mathbf{S} 2$ and $\mathbf{S 3}$, reveal a significantly higher number of intensive reflections (Figure $2 \mathrm{~b}$ and $2 \mathrm{c}$ ). Distinct scattering maxima in small- and wide-angle ranges are evidence for a more ordered polymer packing in the bulk triggered by the post-treatment. Interestingly, all samples exhibit an identical position of the main reflection maxima related to the (100), (010) and (001) crystallographic axes (Figure 3b), indicating that the unit cell dimensions remain almost unaffected by the post-treatment. The corresponding reflections and their parameters for all samples are summarized in Table 1 and will be discussed in more detail in the following. The unchanged position of the interlayer reflection $\left(\mathrm{d}_{100}\right)$ is a result of having two branched chains on the benzodithiophene unit of the polymer, preventing side chain interdigitation and thus defining a minimum interlayer distance. High disordered in the alkyl substituents in $\mathbf{S 1}$ leads to an irregular interlayer spacing and finally to the broadening of the corresponding scattering peak. Similar effects are observed for intralayer distance along (0k0) directions where sterically demanding branched side chains hinder $\pi$-stacking interactions between backbones and induce high disorder in the layer stacks (see definitions for intra- and interlayer in Figure 4a). For this reason for pristine sample S1, the corresponding $\pi$-stacking reflections are missing and only a broad peak is visible in the wide-angle region. The narrowing of diffraction intensities during post-processing (sample $\mathbf{S 2}$ and $\mathbf{S 3}$ ) indicates changes in alkylalkyl interactions between polymer chains improving the long-range interlayer organization. 
Higher order (010) reflections up to $4^{\text {th }}$ orders related to the interlayer and $\pi$-stacking manifest long-range order and increase crystallinity after post-treatment of S2 and S3. Scattering intensities visible for both samples and located at $q_{x y}=1.734 \AA^{-1}$ and $q_{x y}=1.634 \AA^{-1}$ are attributed to the $\pi$-stacking distance of $\mathrm{d}_{\pi-\pi}=0.362 \mathrm{~nm}$ and an intermolecular period of $\mathrm{d}=0.384$ $\mathrm{nm}$ of backbones tilted with respect to the layer axis (Figure 4a). Thereby, the stacking and tilting vector are oriented on the same plane perpendicular to the fiber direction. The relation between both distances of $0.365 \mathrm{~nm}$ and $0.387 \mathrm{~nm}$ determines an in-plane tilting angle of about $\sim 19.0^{\circ}$ between the $\pi$-stacking and the $b$ axis of the unit cell. The reflections on both planes of the patterns in Figure $2 \mathrm{~b}$ and $2 \mathrm{c}$ after post-treatment were assigned by the Miller's indices according to a monoclinic unit cell with parameters of $a=1.66 \mathrm{~nm}, b=1.17 \mathrm{~nm}, c=1.26 \mathrm{~nm}$ and $\gamma=94^{\circ}$ between the $b$ and $a$ axes. The arrangement of the backbones within the lattice is shown in Figure 4a in a top view. The proposed model of the unit cell was confirmed by Cerius ${ }^{2}$ simulations yielding a good overlap between the theoretical and experimental distribution of reflections (Figure 4b). ${ }^{19}$
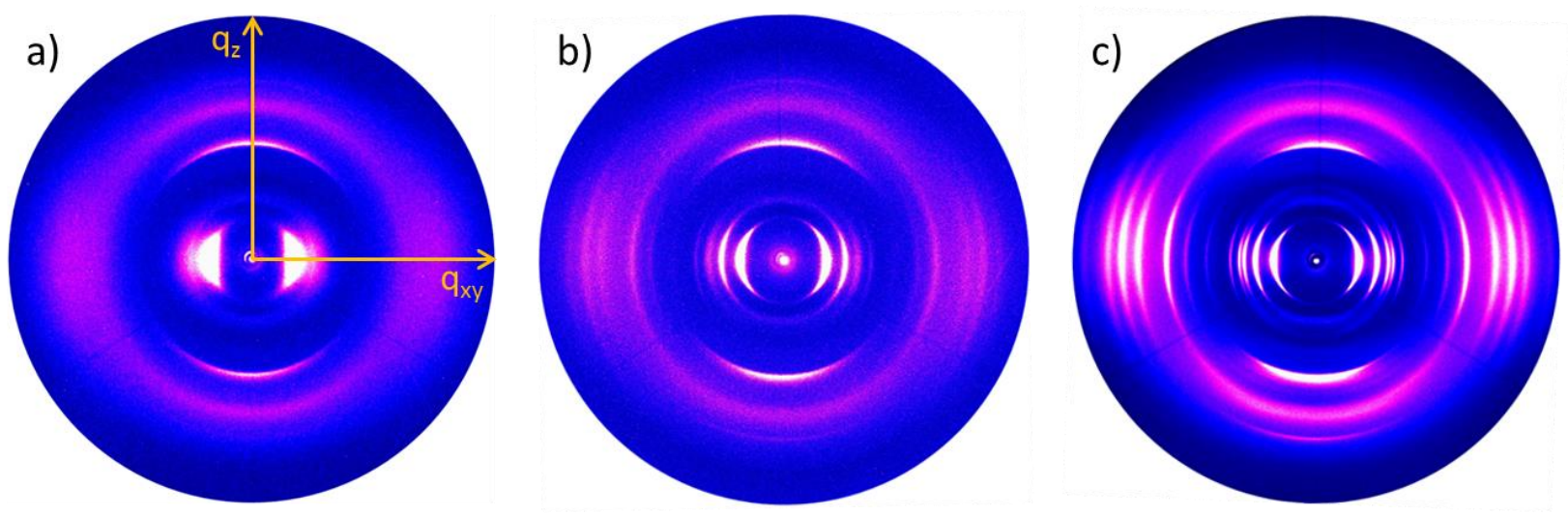

Figure 2. 2DWAXS patterns of a) pristine sample $\mathbf{S 1}\left(\mathrm{q}_{\mathrm{z}}\right.$ and $\mathrm{q}_{\mathrm{xy}}$ directions are valid for all patterns), b)S2 after $48 \mathrm{~h}$ at $120^{\circ} \mathrm{C}$ thermal annealing and c) $\mathbf{S 3}$, after $48 \mathrm{~h}$ solvent vapor annealing at $120^{\circ} \mathrm{C}$. The fiber axis corresponds to the direction of $\mathrm{q}_{\mathrm{z}}$. 

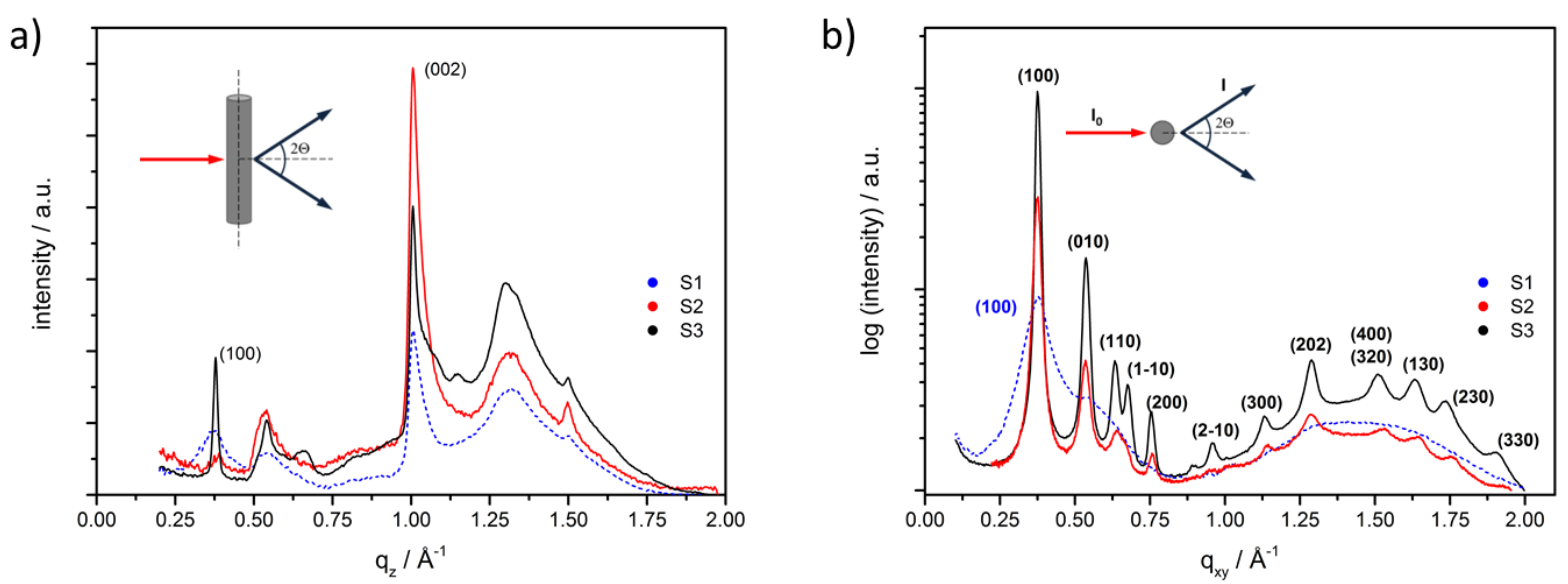

Figure 3. Meridional (a) and equatorial (b) integrations of the 2DWAXS patterns obtained for pristine sample S1 (dashed blue), after thermal annealing S2 (red) and SVA S3 (black). Insets: geometry during the 2DWAXS data acquisition with X-ray scattering in the a) meridional and $b$ ) equatorial direction.

a)

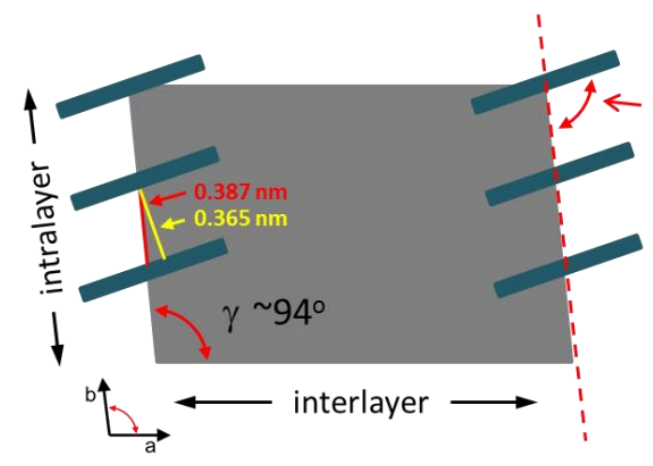

b)

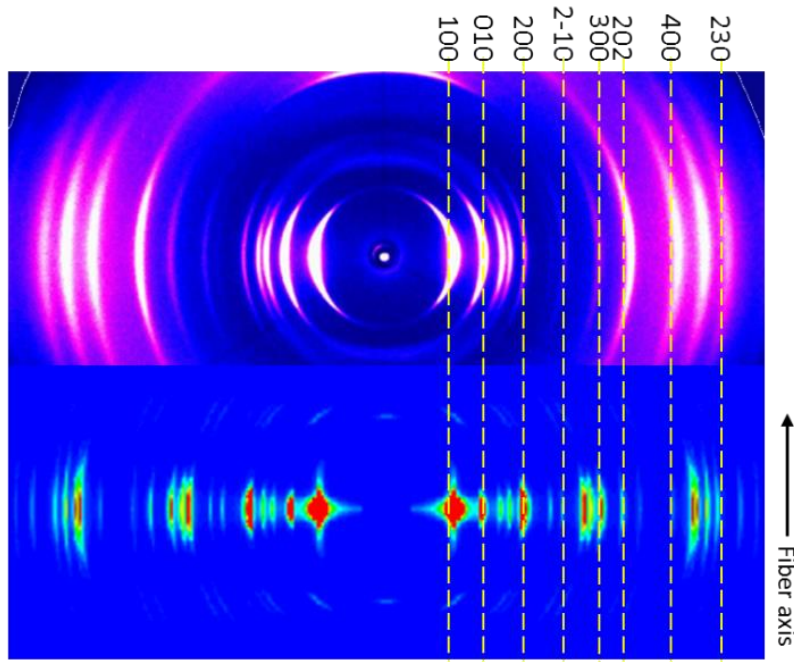

Figure 4. a) Top view of the unit cell of BDT-BTZ present in all three samples S1, S2 and S3 (the blue rectangles represent the polymer backbones, alkyl side chains are omitted, unit cell axes $a$ and $b$ are arranged perpendicular to $c$ which is parallel to the fiber axis); b) comparison of the equatorial part of the experimental 2DWAXS pattern obtained for $\mathbf{S 3}$ (top image) with CERIUS ${ }^{2}$ simulations (bottom image). Dashed lines are eye guides for representative peaks to visualize to good agreement between both patterns.

Isolating and normalizing the diffraction peaks assigned to the inter- (100) and intralayer (010) for each sample allow following the changes of the full width at half maximum (FWHM) of those reflections after post-treatment (Figure 5a). The FWHM $(\Delta q)$ value determines the coherence length $\left(L_{C}\right)$ given by the Scherrer formula $\left(L_{C}=2 \pi / \Delta q\right)$ to estimate the crystal size in 
the bulk and provides quantitative values of the improvement in inter- and intralayer organization. ${ }^{20}$ The FWHM values are compared in Table 1 for the (100) and (010) reflections localized on equatorial plane in small-angle attributed to both in-plane crystallographic axis for different post-treatment procedures. Fitting the pseudo-Voigt function to the experimental data, a close correlation was found between $L_{C}$ and sample treatment. Sample $\mathbf{S 1}$ displays the lowest $L_{C}$ characteristic for a poor polymer order obtained after fiber extrusion. After post-treatment this value increases gradually from $6.87 \mathrm{~nm}$ for $\mathbf{S 1}$ to $26.58 \mathrm{~nm}$ and $40.38 \mathrm{~nm}$ for $\mathbf{S 2}$ and $\mathbf{S 3}$, respectively. This corresponds to average 4, 16 and 24 molecules arranged in one crystalline domain for $\mathbf{S 1}, \mathbf{S} 2$ and $\mathbf{S 3}$ respectively. The coherence lengths calculated along the (0k0) crystal axis reveal an identical trend with values of $3.08 \mathrm{~nm}, 20.58 \mathrm{~nm}$ and $28.38 \mathrm{~nm}(3,51$ and 72 molecules in one domain) for S1, S2 and S3, respectively. This clearly indicates that the thermal and solvent vapor annealing significantly improve the polymer order in both crystallographic directions (h00) and (0k0) and initiate a growth of crystalline domains while at the same time drastically reduce the amorphous/disordered fraction. Interestingly, the two post-treatments have slightly different influence on the order improvements. Conventional thermal annealing enhances mainly the intralayer polymer packing which is initially highly disordered due to sterically demanding branched alkyl chains. On the other hand, solvent vapor used during solvent annealing penetrates the fiber sample and partially re-dissolves the alkylated polymer part inducing a crystallization of the side chains and finally an interlayer long-range order.
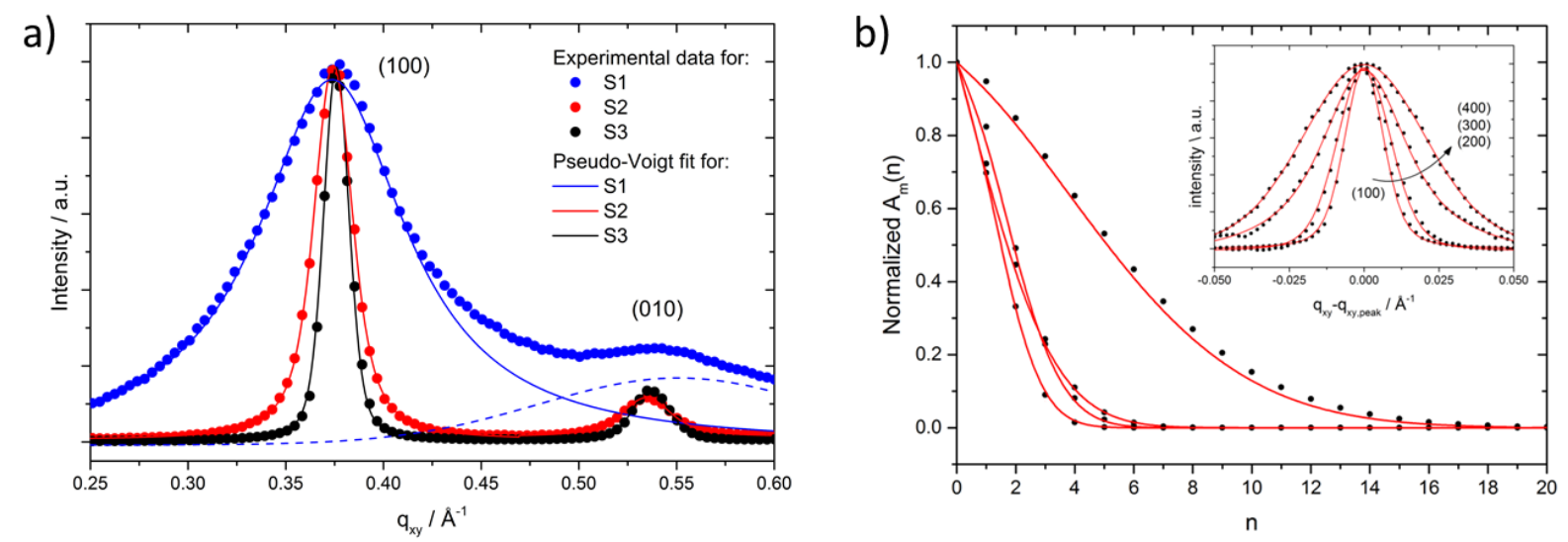

Figure 5. a) 2DWAXS equatorial integration of the small-angle range with the first two reflections related to (100) of the interlayer (continuous line) and (010) for the periods along (0k0) directions (dashed lines). b) The Fourier transforms of the isolated (h00) peaks (dots) with the fit to the Warren-Averbach model (red line) for sample S3. Inset: Isolated and normalized (h00) peaks showing order-dependent broadening (dots - experimental data, red line pseudoVoigt fit function). 
The multiple well-resolved and distinct higher order reflections of $\mathbf{S 2}$ and $\mathbf{S 3}$ (Figure 3b) allow a further quantitative Fourier transform peak shape analysis for determining the average crystallite size, lattice parameter fluctuation, and intracrystalline static disorder. Figure 5b shows isolated and normalized first four diffraction peaks related to the interlayer for S3. The broadening of diffraction peaks is related to dislocations, impurities, chain backbone twists or non-ideal packing what causes an increasing reflection broadening with higher order characteristic for cumulative distortions. ${ }^{21}$ Paracrystalline disorder $(g)$ can be determined experimentally using Xray line-shape analysis to quantitatively extract the fluctuations in molecular spacing due specifically to cumulative disorder in all crystallographic directions. Disorder $g$ is commonly reported as a percent of the mean lattice spacing and for solid materials varies from $0-15 \%$, where $<1 \%$ is indicative for highly crystalline order, $1-10 \%$ represents paracrystallinity, and 10$15 \%$ is considered to be characteristic for a glass or isotropic melt phase. ${ }^{22}$ Hence, $g$ can be used to rank materials quantitatively from perfectly ordered to completely amorphous on a continuous scale. For the analysis of the diffraction line shapes, we used formalism developed by Warren and Averbach and procedures described by Toney and Salleo for determining these values for samples after different post-treatments. ${ }^{22}$

The fitting results are summarized in Table 1 . We found that the average column length (M) for directions along the (h00) axis related to the interlayer exhibits a similar correlation as above described by the coherent length calculated from the Scherrer formula and is slightly increasing from $36.56 \mathrm{~nm}$ to $58.45 \mathrm{~nm}$ for $\mathbf{S 2}$ and $\mathbf{S 3}$, respectively. This trend is in agreement with an increase of domain size along the (h00) crystallographic direction with layers separated by alkyl side chains. The lattice fluctuation parameter $\mathrm{e}_{\mathrm{rms}} \sim 3.2 \pm 0.2 \%$ indicates the variation in $\mathrm{d}$ spacing from one crystallite to another and is similar for both samples $\mathbf{S 2}$ and $\mathbf{S 3}$. This suggests a homogenous growth of the crystalline domains during post-treatment and a homogenous domain size distribution in bulk. Further important information is provided by the paracrystalline disorder (g) which significantly drops from $9.77 \%$ to $6.11 \%$ between sample S2 and S3. These findings show that solvent and thermal annealing impact not only on the texture of the polymer during post-treatment but also improving the crystallinity and intracrystallite order in bulk sample. Exposing the sample to high temperature during thermal $\theta$-annealing increases the local polymer mobility enabling a spatial re-arrangement of the polymer chains and within the layer 
stacks finally forming and expanding larger crystallites. ${ }^{23}$. In comparison to thermal, solvent vapor annealing affects mainly the layer ordering due to penetration of the solvent molecules into the alkyl substituents inducing a crystallization of the side chains. This behavior correlates well with changes of the coherent length calculated for two independent crystallographic directions along the (h00) interlayer and (0k0) intralayer axes. In comparison to thermal post-treatment, solvent annealing generates domains with a 10\% larger coherence length in the h00 direction.

\section{Conclusions}

In summary, we have studied the morphology changes in bulk of a donor-acceptor semiamorphous poly(benzodithiophene-benzotriazole). Annealing at higher temperature or in solvent vapor significantly increased the crystallinity, particularly in the intralayer packing from amorphous to high order including the $\pi$-stacking. The evaluation of the X-ray scattering data allowed the derivation of important structural information like the coherence length and paracrystalinity. These values indicate a different impact of the two annealing procedures on the ordering mechanism. Solvent annealing improved the interlayer order, whereas the polymer packing in the layer structure is enhanced by thermal annealing. Two main conclusions can be drawn from this work. Firstly, initially semi-amorphous conjugated D-A polymers with pronounced $\pi$-stacking disorder can be converted into a higher crystalline structure by posttreatment. Secondly, the insights into the mechanism of the order improvement during posttreatment can be now transferred into thin films for morphology and device optimization. A higher order in polymer packing in the layer structure might favor the charge carrier transport in the $\pi$-stacking direction. On the other hand, the interlayer charge carrier hopping could be facilitated by an increased crystallinity in the layer organization. Both factors are essential for the charge carrier transport in transistors and solar cells.

\section{Experimental methods:}




\section{Solvent Vapor Annealing:}

Solvent Vapor Annealing (SVA) of the extruded BDT-BTZ fiber (sample 3) was performed using 1,2-dichlorobenzene in a solvent reservoir annealing system consisting of a closed chamber of $20 \mathrm{~cm}^{3}$ in volume with a loosely fitted lid that allowed the vapor to leak out slowly. The sample was placed in the chamber and added $2 \mathrm{~cm}^{3}$ of liquid solvent. The thermal solvent vapor annealing was carried out at a temperature of $120{ }^{\circ} \mathrm{C}$. After solvent annealing for $24 \mathrm{~h}$ under an approximately constant vapor atmosphere the fiber was thermal $\theta$-annealed over $20 \mathrm{~min}$ at a temperature $120^{\circ} \mathrm{C}$ to remove residual solvent from the sample.

\section{Thermal Annealing:}

The post-treatment of sample $\mathbf{S 2}$ was performed by thermale-annealing of the extruded fiber at $120^{\circ} \mathrm{C}$ for $48 \mathrm{~h}$ under vacuum.

\section{Two-Dimensional Wide Angle X-Ray Scattering:}

X-ray scattering was performed using a custom setup of a beamline consisting on a Siemens Kristalloflex X-ray source with copper anode X-ray tube operated at $35 \mathrm{kV} / 30 \mathrm{~mA}$ and Osmic confocal MaxFlux optics monochromator. The diffraction beam was collimated with two pinholes (1.2 and $1.0 \mathrm{~mm}$ - Owis, Germany) and anti-scattering pinhole (1.1 mm - Owis, Germany). The patterns were recorded on a MAR345 image plate detector (Marresearch, Germany). The samples were prepared by filament extrusion using a custom-built mini-extruder. Scattering data are expressed as a function of the scattering vector: $q=4 \pi / \lambda \sin (\Theta)$, where $\Theta$ is a half the scattering angle and $\lambda=0.1543 \mathrm{~nm}$ is the wavelength of the incident radiation. Here $q_{x y}$ $\left(q_{z}\right)$ is a component of the scattering vector perpendicular (parallel) to the fiber direction. All Xray scattering measurements were performed under vacuum ( 1mbar) to reduce air scattering and beam damage to the sample. All 2DWAXS data processing and analysis was performed by using the software package Datasqueeze (http://www.datasqueezesoftware.com).

Table 1. Comparison of the peak parameters and Warren-Averbach full fit peak shape analyses. 


\begin{tabular}{|c|c|c|c|}
\hline Sample & S1 & S2 & S3 \\
\hline $\mathrm{q}_{\mathrm{xy}}(100) / \AA^{-1}$ & 0.375 & 0.375 & 0.376 \\
\hline $\mathrm{d}_{100} / \mathrm{nm}$ & 1.68 & 1.68 & 1.67 \\
\hline $\mathrm{FWHM}(100) / \AA^{-1}$ & $0.089 \pm 9.39 * 10^{-4}$ & $0.024 \pm 3.73 * 10^{-5}$ & $0.016 \pm 3.51 * 10^{-5}$ \\
\hline $\mathrm{L}_{\mathrm{C}}(100) / \mathrm{nm}$ & $06.87 \pm 0.07$ & $26.58 \pm 0.04$ & $40.38 \pm 0.09$ \\
\hline $\mathrm{q}_{\mathrm{xy}}(010) / \AA^{-1}$ & 0.553 & 0.535 & 0.537 \\
\hline $\mathrm{d}_{010} / \mathrm{nm}$ & 1.14 & 1.17 & 1.17 \\
\hline $\mathrm{FWHM}(010) / \AA^{-1}$ & $0.204 \pm 3.70 * 10^{-3}$ & $0.031 \pm 6.72 * 10^{-5}$ & $0.022 \pm 3.73 * 10^{-5}$ \\
\hline $\mathrm{L}_{\mathrm{C}}(010) / \mathrm{nm}$ & $03.08 \pm 0.06$ & $20.58 \pm 0.05$ & $28.38 \pm 0.05$ \\
\hline $\mathrm{M}(100) / \mathrm{nm}$ & n.a. & $36.56 \pm 0.12$ & $58.45 \pm 0.47$ \\
\hline $\mathrm{G}(100) / \%$ & n.a. & $9.77 \pm 4.69$ & $6.11 \pm 2.97$ \\
\hline $\mathrm{e}_{\mathrm{rms}}(100) / \%$ & n.a. & $3.1 \pm 0.1$ & $3.2 \pm 0.2$ \\
\hline
\end{tabular}

FWHM - full width at half maximum, $\mathrm{L}_{\mathrm{C}}$ - coherence length, $\mathrm{M}$ - average column size, g - paracrystalline disorder, $\mathrm{e}_{\mathrm{rms}}$ - lattice fluctuation parameter.

1. Facchetti, A., $\pi$-Conjugated Polymers for Organic Electronics and Photovoltaic Cell Applications $\uparrow$. Chemistry of Materials 2011, 23 (3), 733-758.

2. $\quad$ Guo, X.; Baumgarten, M.; Müllen, K., Designing $\pi$-conjugated polymers for organic electronics. Progress in Polymer Science 2013, 38 (12), 1832-1908.

3. Mullen, K.; Pisula, W., Donor-Acceptor Polymers. J Am Chem Soc 2015, 137 (30), 95035.

4. $\quad$ Kang, H.; Kim, K.-H.; Choi, J.; Lee, C.; Kim, B. J., High-Performance All-Polymer Solar Cells Based on Face-On Stacked Polymer Blends with Low Interfacial Tension. ACS Macro Letters 2014, 3 (10), 1009-1014.

5. Zhou, E.; Nakano, M.; Izawa, S.; Cong, J.; Osaka, I.; Takimiya, K.; Tajima, K., AllPolymer Solar Cell with High Near-Infrared Response Based on a Naphthodithiophene Diimide (NDTI) Copolymer. ACS Macro Letters 2014, 3 (9), 872-875.

6. (a) Biniek, L.; Schroeder, B. C.; Nielsen, C. B.; McCulloch, I., Recent advances in high mobility donor-acceptor semiconducting polymers. Journal of Materials Chemistry 2012, 22 (30), 14803; (b) Kang, I.; Yun, H. J.; Chung, D. S.; Kwon, S. K.; Kim, Y. H., Record high hole mobility in polymer semiconductors via side-chain engineering. J Am Chem Soc 2013, 135 (40), 14896-9; (c) Sun, B.; Hong, W.; Yan, Z.; Aziz, H.; Li, Y., Record high electron mobility of 6.3 
$\mathrm{cm}(2) \mathrm{V}(-)(1) \mathrm{s}(-)(1)$ achieved for polymer semiconductors using a new building block. $A d v$ Mater 2014, 26 (17), 2636-42, 2613.

7. (a) Olivier, Y.; Niedzialek, D.; Lemaur, V.; Pisula, W.; Mullen, K.; Koldemir, U.; Reynolds, J. R.; Lazzaroni, R.; Cornil, J.; Beljonne, D., 25th anniversary article: high-mobility hole and electron transport conjugated polymers: how structure defines function. Adv Mater 2014, 26 (14), 2119-36; (b) Pisula, W.; Tsao, H.; Dudenko, D.; Cho, D.; Puniredd, S.; Zhao, Y.; Mavrinskiy, A.; Shu, J.; Hansen, M.; Baumgarten, M.; Müllen, K., Solid-State Organization and Ambipolar Field-Effect Transistors of Benzothiadiazole-Cyclopentadithiophene Copolymer with Long Branched Alkyl Side Chains. Polymers 2013, 5 (2), 833-846.

8. Mei, J.; Bao, Z., Side Chain Engineering in Solution-Processable Conjugated Polymers. Chemistry of Materials 2014, 26 (1), 604-615.

9. (a) Lee, J. B.; Kim, K. H.; Hong, C. S.; Choi, D. H., High-performance amorphous donor-acceptor conjugated polymers containing $\mathrm{X}$-shaped anthracene-based monomer and 2,5bis(2-octyldodecyl)pyrrolo[3,4-c]pyrrole-1,4(2H,5H)-dione for organic thin-film transistors. Journal of Polymer Science Part A: Polymer Chemistry 2012, 50 (14), 2809-2818; (b) Zhang, W.; Smith, J.; Watkins, S. E.; Gysel, R.; McGehee, M.; Salleo, A.; Kirkpatrick, J.; Ashraf, S.; Anthopoulos, T.; Heeney, M.; McCulloch, I., Indacenodithiophene semiconducting polymers for high-performance, air-stable transistors. J Am Chem Soc 2010, 132 (33), 11437-9; (c) Liu, J.; Zhang, R.; Sauve, G.; Kowalewski, T.; McCullough, R. D., Highly disordered polymer field effect transistors: N-alkyl dithieno[3,2-b:2',3'-d]pyrrole-based copolymers with surprisingly high charge carrier mobilities. J Am Chem Soc 2008, 130 (39), 13167-76.

10. Noriega, R.; Rivnay, J.; Vandewal, K.; Koch, F. P.; Stingelin, N.; Smith, P.; Toney, M. F.; Salleo, A., A general relationship between disorder, aggregation and charge transport in conjugated polymers. Nat Mater 2013, 12 (11), 1038-44.

11. (a) Banal, J. L.; Subbiah, J.; Graham, H.; Lee, J.-K.; Ghiggino, K. P.; Wong, W. W. H., Electron deficient conjugated polymers based on benzotriazole. Polym. Chem. 2013, 4 (4), $1077-$ 1083; (b) Lee, J. K.; Gwinner, M. C.; Berger, R.; Newby, C.; Zentel, R.; Friend, R. H.; Sirringhaus, H.; Ober, C. K., High-performance electron-transporting polymers derived from a heteroaryl bis(trifluoroborate). J Am Chem Soc 2011, 133 (26), 9949-51.

12. Wong, W. W. H.; Subbiah, J.; Puniredd, S. R.; Pisula, W.; Jones, D. J.; Holmes, A. B., Benzotriazole-based donor-acceptor conjugated polymers with a broad absorption in the visible range. Polym. Chem. 2014, 5 (4), 1258-1263.

13. (a) Chavis, M. A.; Smilgies, D. M.; Wiesner, U. B.; Ober, C. K., Widely Tunable Morphologies in Block Copolymer Thin Films Through Solvent Vapor Annealing Using Mixtures of Selective Solvents. Adv Funct Mater 2015, 25 (20), 3057-3065; (b) Luo, M.; Scott, D. M.; Epps, T. H., Writing Highly Ordered Macroscopic Patterns in Cylindrical Block Polymer Thin Films via Raster Solvent Vapor Annealing and Soft Shear. Acs Macro Letters 2015, 4 (5), 516-520.

14. Gholamkhass, B.; Servati, P., Solvent-vapor induced morphology reconstruction for efficient PCDTBT based polymer solar cells. Organic Electronics 2013, 14 (9), 2278-2283.

15. Tang, H.; Lu, G.; Li, L.; Li, J.; Wang, Y.; Yang, X., Precise construction of PCBM aggregates for polymer solar cellsvia multi-step controlled solvent vapor annealing. J. Mater. Chem. 2010, 20 (4), 683-688.

16. Wei, G.; Wang, S.; Sun, K.; Thompson, M. E.; Forrest, S. R., Solvent-Annealed Crystalline Squaraine: PC70BM (1:6) Solar Cells. Advanced Energy Materials 2011, 1 (2), 184187. 
17. Yuan, Q.; Mannsfeld, S. C. B.; Tang, M. L.; Roberts, M.; Toney, M. F.; DeLongchamp, D. M.; Bao, Z., Microstructure of Oligofluorene Asymmetric Derivatives in Organic Thin Film Transistors. Chemistry of Materials 2008, 20 (8), 2763-2772.

18. Beaujuge, P. M.; Tsao, H. N.; Hansen, M. R.; Amb, C. M.; Risko, C.; Subbiah, J.; Choudhury, K. R.; Mavrinskiy, A.; Pisula, W.; Bredas, J. L.; So, F.; Mullen, K.; Reynolds, J. R., Synthetic principles directing charge transport in low-band-gap dithienosilole-benzothiadiazole copolymers. J Am Chem Soc 2012, 134 (21), 8944-57.

19. (a) Kumar, P.; Shivananda, K. N.; Zajączkowski, W.; Pisula, W.; Eichen, Y.; Tessler, N., The Relation Between Molecular Packing or Morphology and Chemical Structure or Processing Conditions: the Effect on Electronic Properties. Adv Funct Mater 2014, 24 (17), 2530-2536; (b) Cerius $^{2}$ Cerius $^{2}$, Accelrys: Inc.: San Diego, 1998.

20. (a) Scardi, P.; Leoni, M.; Delhez, R., Line broadening analysis using integral breadth methods: a critical review. Journal of Applied Crystallography 2004, 37 (3), 381-390; (b) Balzar, D.; Audebrand, N.; Daymond, M. R.; Fitch, A.; Hewat, A.; Langford, J. I.; Le Bail, A.; Louër, D.; Masson, O.; McCowan, C. N.; Popa, N. C.; Stephens, P. W.; Toby, B. H., Size-strain linebroadening analysis of the ceria round-robin sample. Journal of Applied Crystallography 2004, 37 (6), 911-924.

21. Prosa, T. J.; Moulton, J.; Heeger, A. J.; Winokur, M. J., Diffraction Line-Shape Analysis of Poly(3-dodecylthiophene): A Study of Layer Disorder through the Liquid Crystalline Polymer Transition. Macromolecules 1999, 32 (12), 4000-4009.

22. (a) Rivnay, J.; Noriega, R.; Kline, R. J.; Salleo, A.; Toney, M. F., Quantitative analysis of lattice disorder and crystallite size in organic semiconductor thin films. Physical Review B 2011, 84 (4); (b) Rivnay, J.; Noriega, R.; Northrup, J. E.; Kline, R. J.; Toney, M. F.; Salleo, A., Structural origin of gap states in semicrystalline polymers and the implications for charge transport. Physical Review B 2011, 83 (12).

23. Campoy-Quiles, M.; Ferenczi, T.; Agostinelli, T.; Etchegoin, P. G.; Kim, Y.; Anthopoulos, T. D.; Stavrinou, P. N.; Bradley, D. D.; Nelson, J., Morphology evolution via selforganization and lateral and vertical diffusion in polymer:fullerene solar cell blends. Nat Mater 2008, 7 (2), 158-64. 